\title{
Noncompaction of ventricular myocardium in an elderly woman
}

\author{
Alessio Currò · Alessandro Lauro · Raffaella Gallo • \\ Andrea Corsonello
}

Received: 20 July 2009/Accepted: 24 August 2009/Published online: 12 September 2009

(C) SIMI 2009

Noncompaction of ventricular myocardium (NVM) is classified among genetic cardiomyopathies, and is thought to be caused by failure of the myocardial compaction process during embryogenesis [1]. This leads to a hypertrabeculated left ventricular myocardium with deep intertrabecular recesses that communicate directly with the main ventricular chamber [2]. In this paper, we describe a case of NVM in an elderly patient.

A 68-year-old woman was admitted to our Cardiology unit because of worsening dyspnea and peripheral edema. Eight years prior to admission, she had been diagnosed with hypertension and persistent atrial fibrillation, and was placed on warfarin therapy. Five years later, the patient was acutely admitted for dyspnea, and during the stay she had a dysrhythmic syncopal episode. On this occasion, after an extensive diagnostic work-up including coronary angiography, which revealed a normal coronary artery tree, she underwent the implantation of a biventricular pace-maker and cardioverter defibrillator, and was discharged with a diagnosis of dilated cardiomyopathy. At the time of the present admission, the patient was taking warfarin, furosemide $50 \mathrm{mg} /$ day, ramipril $5 \mathrm{mg} /$ day, digoxin $0.125 \mathrm{mg} /$ day, and bisoprolol $1.25 \mathrm{mg} /$ day. The patient was in NYHA class IV, and on physical examination, she appeared acutely ill. She had a prominent jugular pulse, a holosystolic murmur over the mitral and aortic valves, reduced breath sounds and rales at both pulmonary bases,

A. Currò · A. Lauro · R. Gallo · A. Corsonello Istituto Nazionale di Ricovero e Cura per Anziani (INRCA), Cosenza, Italy

A. Corsonello $(\square)$

Viale della Resistenza Pal. Alfa Scala H,

87036 Rende (CS), Italy

e-mail: andrea_corsonello@tin.it and peripheral leg edema. The ECG confirmed the presence of atrial fibrillation and left bundle branch block. A chest $\mathrm{X}$-ray study revealed an enlarged cardiac silhouette and hilar congestion. Admission blood analyses were normal except for a plasma cholesterol $(267 \mathrm{mg} / \mathrm{dl})$ and pro-BNP (1,463 pg/ml, normal range 0-450). Echocardiographic examination revealed a dilated and globus left ventricle (tele-diastolic diameter $60 \mathrm{~mm}$ ) with diffuse hypokinesia (ejection fraction 25\%), bilateral atrial dilatation, moderate mitral and mild tricuspidal valve insufficiencies with an estimated systolic pulmonary pressure $55 \mathrm{mmHg}$, and diastolic dysfunction (E/A ratio $>2$ ). In the lateral wall and apical region, several trabeculations, typical of NVM, were observed (Fig. 1). The ratio between noncompacted and compacted myocardium in telediastole was 2.3. No other valvular abnormalities were observed. After two days of therapy with furosemide $40 \mathrm{mg}$ i.v. bid the clinical condition improved, and the patient's medications were raised to ramipril to $10 \mathrm{mg} /$ day, bisoprolol to $5 \mathrm{mg} /$ day, and oral furosemide to $100 \mathrm{mg} /$ day, while continuing therapy with warfarin and digoxin, and starting therapy with lovastatin $20 \mathrm{mg} /$ day. The patient was discharged and followed-up for 6 months, and did not experience any further heart failure exacerbations. Her two sons agreed to be examined by echocardiographic screening, and were found to be free of NVM.

The NVM is a rare congenital cardiomyopathy, which can be sporadic or familial [2,3]. Although initially described in children, several case series report this entity to also be present in adults, but rarely, in elderly patients [2-4]. The prevalence of NVM is estimated to range between $0.05 \%$ and $0.24 \%$ of all echocardiographic investigations in recent studies, while its incidence in the general population is yet unknown [2,3]. Patients carrying NVM can remain asymptomatic or with only minimal 

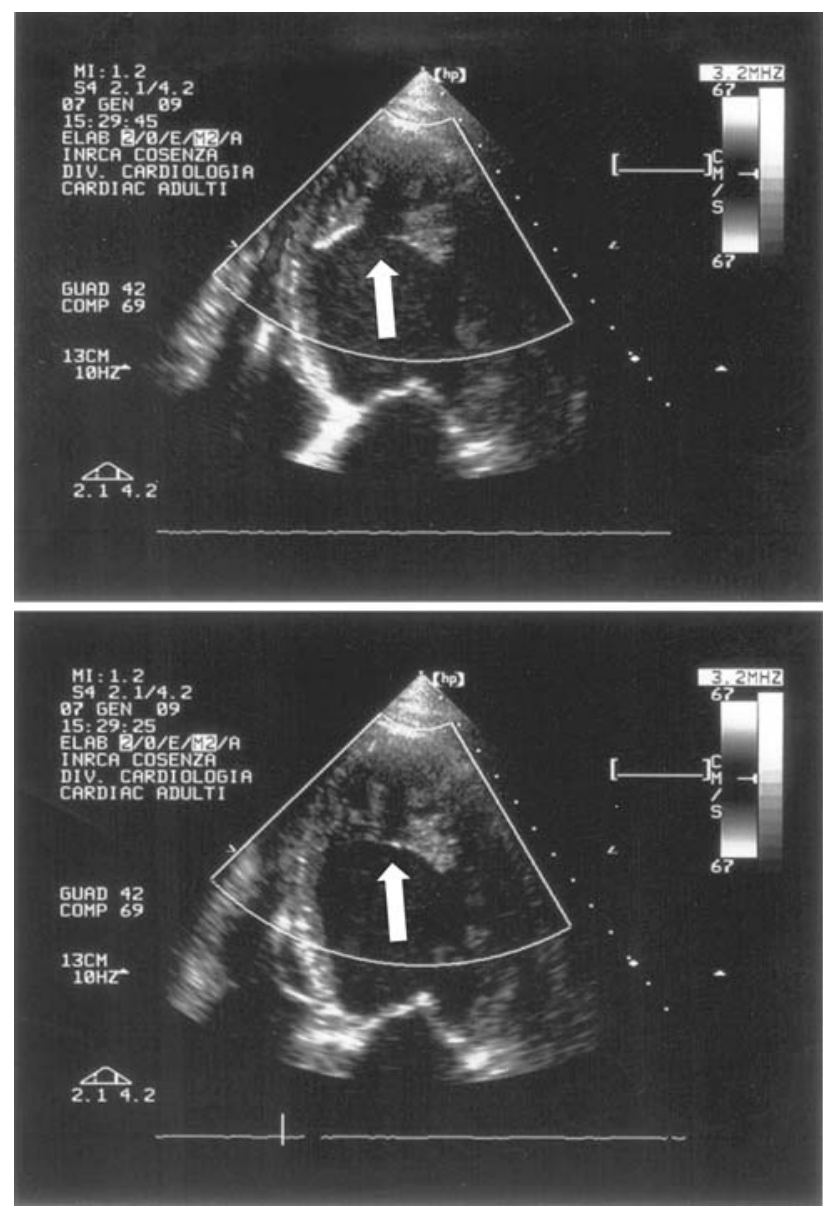

Fig. 1 Echocardiography showing hypertrabeculation (arrows) of the left ventricle lateral wall and apical region

symptoms for many years, but the reasons explaining the delayed onset of symptoms until an older age in some NVM patients remains to be elucidated. The classic clinical manifestations include heart failure, dysrhythmias, and thromboembolic events $[2,3]$. As regards the therapeutic approach to these patients, the limited amount of available research suggests that the most reasonable approach is to use standard treatment for the general conditions that develop from NVM, including heart failure, dysrhythmias, and thromboembolic events [2,3]. Since finding innovative and effective treatments for rare medical disorders is always a challenge, increased awareness by health care providers worldwide could help to identify a greater number of cases, to start appropriate treatments and, eventually, to find new therapeutic strategies.

The most widely accepted method of diagnosing NVM is by echocardiography, and a recognized set of diagnostic criteria is available[5]: (1) $>3$ prominent trabeculations protruding from the left ventricular wall with deep intertrabecular recesses; (2) direct blood flow from the ventricular cavity into the intertrabecular recesses as visualized on color Doppler; (3) absence of any coexisting cardiac anomalies; and (4) ratio of noncompacted subendocardial layer to compacted subepicardial layer at endsystole $\geq 2$ [5]. However, diagnosis by echocardiography is operator dependent, and requires attentive and experienced providers to ensure these patients are not misdiagnosed as having another form of cardiomyopathy, or are missed altogether [2,3].

The clinical history of our patient was characterized by two of the three most frequent clinical manifestations of NVM (i.e. heart failure and dysrhythmias), and highlights the risk of misdiagnosis. Indeed, it is conceivable that accurate echocardiographic examination would have revealed the presence of left ventricle hypertrabeculation at the time of the original diagnosis of hypertension and persistent atrial fibrillation, or when the episode of dysrhythmic syncope occurred. Alternatively, we cannot rule out that such an echocardiographic finding, appearing the same as the congenital disease, might be acquired from heart disease occurring in later life. In this case, its clinical and prognostic implications would remain to be established. For all the above reasons, besides presenting a clinical entity that is rarely observed in the elderly [4], our case report underlines the importance of making echocardiographers more familiar with this condition when examining older patients.

Conflict of interest statement The authors declare that they have no conflict of interest related to the publication of this manuscript.

\section{References}

1. Maron BJ, Towbin JA, Thiene G et al (2006) Contemporary definitions and classification of the cardiomyopathies: an American Heart Association Scientific Statement from the Council on Clinical Cardiology, Heart Failure and Transplantation Committee; Quality of Care and Outcomes Research and Functional Genomics and Translational Biology Interdisciplinary Working Groups; and Council on Epidemiology and Prevention. Circulation 113:1807-1816

2. Eilen D, Peterson N, Karkut C, Movahed A (2008) Isolated noncompaction of the left ventricular myocardium: a case report and literature review. Echocardiography 25:755-761

3. Song ZZ (2008) Echocardiography in the diagnosis left ventricular noncompaction. Cardiovasc Ultrasound 6:64

4. Khan IA, Biddle WP, Najeed SA et al (2003) Isolated noncompaction cardiomyopathy presenting with paroxysmal supraventricular tachycardia-case report and literature review. Angiology $54: 243-250$

5. Jenni R, Oechslin E, Schneider J, Attenhofer Jost C, Kaufmann PA (2001) Echocardiographic and pathoanatomical characteristics of isolated left ventricular non-compaction: a step towards classification as a distinct cardiomyopathy. Heart 86:666-671 